\section{AB0337 ASSESSMENT OF NUTRITIONAL STATUS IN WOMEN WITH RHEUMATOID ARTHRITIS MEASURED BY DUAL ENERGY X-RAY ABSORPTIOMETRY}

D. Reina ${ }^{1}$, C. Gómez Vaquero ${ }^{2}$, J.M. Nolla ${ }^{2} .{ }^{1}$ Rheumatology, Consorci Sanitari Integral; ${ }^{2}$ Rheumatology, Hospital Universitari de Bellvitge, barcelona, Spain

Background: As many other chronic diseases, rheumatoid arthritis (RA) has been related to an impairment of the nutritional status of multifactorial etiology. Even if Body Mass Index (BMI) has traditionally been used, it is not always a valid method, and there are still lots of questions without answer in how to evaluate ideally the nutritional status in these patients. Only very few studies had evaluated it by Dual Energy X Ray Absorptiometry (DXA).

Objectives: 1) To evaluate the nutritional status in women with RA and to compare with a population of same age women without RA using DXA for the analysis of whole body composition, 2) differences between the prevalence of alterations of the nutritional status measured by DXA and by the classical methods used in clinical care, 3) relation of RA time of evolution, inflammatory activity, physical function and dietary intake on altered nutritional status evaluated by DXA.

Methods: Case-control study including 89 patients that were diagnosed with RA and a control group (100) composed by patients affected by other non-inflammatory rheumatic diseases as soft tissue diseases. All the clinical charts were revised in order to record the following data: age, BMI, RA duration, history, activity and disability, serum albumin, Dietary intake, Whole body DXA assessment and Skeletal muscle index (SMI).

Results: Mean age of patients was $62 \pm 8$ years. Mean duration of RA was 13.7 \pm 9.3 years. Mean DAS28 was $3.7 \pm 1.4$ and mean Health Assessment Questionnaire was $0.88 \pm 0.77$. BMl of the patients was $27.43 \pm 5.16$ and $27.78 \pm 3.98$ in controls ( $p$ : ns). Albumin was within normal range in all patients.

RA patients presented a statistically significant lower lean mass than controls in all locations and lower fat mass in limbs, along with a higher fat trunk.

RA duration was found to be inversely correlated to BMI and lean mass and directly correlated with fat mass.

Neither BMI nor albumin correlated with DXA parameters.

RA patients fulfilled criteria of sarcopenia in $44 \%$ of de cases vs $19 \%$ of controls $(p<0,001)$. In RA patients, regarding SMI, BMI showed a high specificity to detect sarcopenia (94\% of the patients with low BMI had sarcopenia) but low sensitivity ( $47 \%$ of the patients with normal or overweight BMI had sarcopenia).

Conclusions: RA patients have an impairment of nutritional status associated to time of evolution that resembles sarcopenia and that is not predicted by BMI.

Disclosure of Interest: None declared

DOI: 10.1136/annrheumdis-2018-eular.5330

\section{AB0338 CLINICAL CHARACTERISTICS OF PATIENTS WITH RHEUMATOID ARTHRITIS WHO USED COMPUTER TERMINALS FOR SELF-ASSESSMENT OF DISEASE ACTIVITY AND QUALITY OF LIFE}

E. Pogozheva, V. Amirdzhanova. V. A. Nasonova Research Institute of Rheumatology, Moscow, Russian Federation

Objectives: To present the clinical features, pharmacotherapy, activity and quality of life in pts with rheumatoid arthritis (RA) who used computer terminals for selfassessment of pts with rheumatic diseases.

Methods: The study included 976 RA patients from the cohort of "TERMINAL" multicenter study, envisaging pts' self-assessment of disease activity and quality of life using a designated computer system ("Computer terminals for self-assessment of pts with rheumatic diseases) before the visit to the rheumatologist. Mean age $52,3 \pm 13,3$ years, mean disease duration $10,2 \pm 8,5$ years, $85 \%$ female. Baseline clinical parameters and pharmacotherapy were assessed, as well as disease activity (using DAS 28 and RAPID-3), and quality of life (using HAQ and EQ-5D). Results: $83 \%$ of pts were RF positive, and $60 \%$ ACCP positive. Almost all $(91,2 \%)$ pts received conventional DMARDs. $70,2 \%$ of them received methotrexate: $56,7 \% 15 \mathrm{mg} /$ week and $13,5 \%>15 \mathrm{mg} /$ week (from 17,5 to $40 \mathrm{mg} /$ week). $20,5 \%$ pts were treated earlier with low doses of glucocorticoids. $6,6 \%$ pts received Infliximab, 16,4\% - Rituximab and 9,8\% pts were treated with more than 1 biological agent in anamnesis. $46,2 \%$ of pts had high disease activity, $40,9 \%$ moderate, $4,5 \%$ low and $1,5 \%$ pts achieved clinical remission (mean DAS28 score $4,2 \pm 1,8$, RAPID $13,6 \pm 3,6$ ). Only $14,3 \%$ pts achieved general population HAQ values. The remaining showed significant reduction in the quality of life (mean HAQ $1,7 \pm 0,9, E Q-5 D 0,6 \pm 0,2) .7,4 \%$ of the pts had prosthetic joints.

Conclusions: In the studied population almost all patients were treated with DMARDs (mostly methotrexate), and about $30 \%$ received biological agents. High to moderate disease activity and reduction in quality of life were typical for this population. Persistence of disease activity and functional insufficiency in pts who received DMARD therapy may be explained by poor monitoring in real clinical practice. The introduction of computer system for self-assessment of disease activity and quality of life in the out-patient facilities will improve the interaction between doctors, nurses and patients, providing better control of therapy efficacy in RA patients.

Disclosure of Interest: None declared

DOI: 10.1136/annrheumdis-2018-eular.3256

\section{AB0339 SARCOPENIA AND EARLY FRAILTY SYNDROME IN RHEUMATOID ARTHRITIS}

E. Trujillo ${ }^{1}$, M.R. Garcia-Marrero ${ }^{2}$, M.I. Fuentes ${ }^{2}$, M.D.M. Trujillo ${ }^{3} .{ }^{1}$ Servicio de Reumatologia, Hospital Universitario de Canarias, la laguna; ${ }^{2}$ Gerencia de Atención Primaria, Tenerife; ${ }^{3}$ Funcanis, Fundación Canaria de Investigación, Canarias, Spain

Background: Sarcopenia and frailty are common in older persons and pose particular challenges for health and social care systems.

Sarcopenia, the loss of skeletal muscle mass, is a core component of physical frailty that together impact negatively on an individual's capability to live independently.

Frailty is defined as a syndrome of physiological decline in late life, characterised by marked vulnerability to adverse health outcomes. Frail adults are less able to adapt to stressors such as acute illness or trauma than non-frail adults. This increased vulnerability contributes to increased risk for multiple adverse outcomes, including procedural complications, falls, institutionalisation, disability, and death.

Rheumatoid arthritis (RA) is a chronic disabling disease, which leads to functional limitations and diminishes health-related quality of life. The presence of comorbidity and polypharmacy are both related to RA severity.

Objectives: The aim of this study was to assess the prevalence of sarcopenia and frailty syndrome in patients with RA.

Methods: Cross-sectional, observational and descriptive study in patients with RA (ACR criteria) older than 50 years.

Sarcopenia was defined as per the European Working Group on Sarcopenia in Older People definition as Skeletal muscle mass index (SMI) $\leq 8.87 \mathrm{~kg} / \mathrm{m}^{2}$ in men and $\leq 6.42 \mathrm{~kg} / \mathrm{m}^{2}$ in women. Body composition analysis was performed using bioelectrical impedance analysis (BIA)

Fragility was measured according to the 5 criteria proposed by Fried, using the Frail scale, and it was considered fragile to the patient who met at least 3 and prefragiles to those who met at least 2.

Frail scale: Based on five items, reflecting performance, selfreports and common co-morbidities (Morley JE et al. J Nutr Health Ageing 2012;16(7):601-8).

FRAIL SCALE

Did you feel worn out? or Did you feel tired?

Ability to climb one flight of stairs

Ability to walk $100 \mathrm{~m}$

Self-report of $>5 \%$ wt loss

$\leq 5$ of: dementia; heart Disease; depression; arthritis; asthma; bronchitis/emphy sema; diabetes; hypertension; osteoporosis; stroke.

Results: 283 consecutive RA patients were included, $83.4 \%$ ) were female. Mean age was 63.3 years and mean disease duration was 10.4 years.

Mean number of comorbidities was 1.48 , with systemic hypertension and obesity as the most frequent ones (33.8\% and $26.4 \%$, respectively). Polypharmacy was found in $96.8 \%$ and $64.7 \%$ received more than five drugs simultaneously.

$31 \%$ presented some degree of sarcopenia. $21.5 \%$ met frailty criteria $(42 \%$ in $\geq 65$ years old patients).

Conclusions: Prevalence of sarcopenia and frailty syndrome in this study was high. Rheumatologists should make an early detection of signs of frailty.

The screening and early detection of frailty can spur reforms to make routine care less hazardous, can focus on outcomes most relevant to patients and can aid in understanding effectiveness of health care interventions, including at the population level.

Disclosure of Interest: None declared

DOI: 10.1136/annrheumdis-2018-eular.3519

\section{AB0340 ASSOCIATION BETWEEN MICROALBUMINURIA AND METABOLIC SYNDROME IN PATIENTS WITH RHEUMATOID ARTHRITIS}

E. Baraka ${ }^{1}$, S. Abdel Moneim ${ }^{1}$, R. El Tanawy ${ }^{1}$, A. Shoshan ${ }^{1}$, Y. Ismail ${ }^{2}$.

${ }^{1}$ Rheumatology, Rehabilitation and Physical medicine; ${ }^{2}$ Clinical pathology, Benha Faculty of Medicine, Benha University, Cairo, Egypt

Background: Rheumatoid arthritis (RA) is an autoimmune, symmetrical polyarticular disease characterised by chronic inflammation of the synovial joints. Microalbuminuria (MA) occurs as a leakage of small amounts of albumin into the urine Metabolic syndrome (MetS) describes risk factors for cardiovascular diseases such as dyslipidaemia, obesity, hypertension and diabetes. 